\title{
Expert Elicitation as a Means to Attribute 28 Enteric Pathogens to Foodborne, Waterborne, Animal Contact, and Person-to-Person Transmission Routes in Canada
}

\author{
Ainslie J. Butler, M. Kate Thomas, and Katarina D.M. Pintar
}

\begin{abstract}
Enteric illness contributes to a significant burden of illness in Canada and globally. Understanding its sources is a critical step in identifying and preventing health risks. Expert elicitation is a powerful tool, used previously, to obtain information about enteric illness source attribution where information is difficult or expensive to obtain. Thirty-one experts estimated transmission of 28 pathogens via major transmission routes (foodborne, waterborne, animal contact, person-to-person, and other) at the point of consumption. The elicitation consisted of a (snowball) recruitment phase; administration of a pre-survey to collect background information, an introductory webinar, an elicitation survey, a 1day discussion, survey readministration, and a feedback exercise, and surveys were administered online. Experts were prompted to quantify changes in contamination at the point of entry into the kitchen versus point of consumption. Estimates were combined via triangular probability distributions, and medians and $90 \%$ credible-interval estimates were produced. Transmission was attributed primarily to food for Bacillus cereus, Clostridium perfringens, Cyclospora cayetanensis, Trichinella spp., all three Vibrio spp. categories explored, and Yersinia enterocolitica. Multisource pathogens (e.g., transmitted commonly through both water and food) such as Campylobacter spp., four Escherichia coli categories, Listeria monocytogenes, Salmonella spp., and Staphylococcus aureus were also estimated as mostly foodborne. Water was the primary pathway for Giardia spp. and Cryptosporidium spp., and person-to-person transmission dominated for six enteric viruses and Shigella spp. Consideration of the point of attribution highlighted the importance of food handling and cross-contamination in the transmission pathway. This study provides source attribution estimates of enteric illness for Canada, considering all possible transmission routes. Further research is necessary to improve our understanding of poorly characterized pathogens such as sapovirus and E. coli subgroups in Canada.
\end{abstract}

\section{Introduction}

$\mathbf{E}$ NTERIC ILLNESS IS A SIGNIFICANT public health concern both in Canada and globally (Thomas et al., 2013). It is estimated that 20.5 million $(90 \%$ credible interval [CrI]: 19.3-21.7 million) cases of enteric illness occur in Canada annually (Thomas et al., 2013). Source attribution refers to the proportioning of illness to sources and transmission routes. Current source attribution methods for infectious enteric illness include the following: comparative exposure assessments, analysis of outbreak data, case-control studies, intervention studies, microbial subtype modeling, and expert elicitation (Pires, 2013). Expert elicitation enables exploration of research questions and the associated uncertainty for issues where data are expensive to obtain or otherwise unavailable (e.g., lack of national Bacillus cereus surveillance data in Canada, inconsistent follow-up and reporting of exposure factors associated with reportable enteric diseases) (Pires, 2013; Butler et al., 2015; in press). Expert elicitation for enteric illness source attribution has been undertaken in New Zealand (Cressey and Lake, 2005), the United States (Hoffmann et al., 2006), the Netherlands (Havelaar et al., 2008), Canada (Ravel et al., 2010; Davidson et al., 2011), and Australia (Vally et al., 2014).

Because enteric illnesses are not solely foodborne (Pires, 2013), it is important to focus on the whole spectrum of enteric disease transmission to inform prevention. As part of burden of illness and source attribution work of the Public Health Agency of Canada (PHAC), an expert elicitation to attribute enteric diseases to their respective transmission

Centre for Foodborne, Environmental, and Zoonotic Infectious Diseases, Public Health Agency of Canada, Ottawa, Ontario, Canada.

(C) The Author(s) 2015; Published by Mary Ann Liebert, Inc. This Open Access article is distributed under the terms of the Creative Commons License (http://creativecommons.org/licenses/by/4.0), which permits unrestricted use, distribution, and reproduction in any medium, provided the original work is properly credited. 
routes in Canada was completed. This study aims to improve the understanding of the relative role of transmission pathways in the burden of enteric illness, focusing on 28 pathogens of public health importance in Canada (Thomas et al., 2013). A secondary objective was to consider the role of cross-contamination in the kitchen, through consideration of attribution at the point of entry into the kitchen versus contamination at the point of consumption. These estimates can be used to guide future research and surveillance efforts.

\section{Materials and Methods}

A 6-stage expert elicitation was designed to produce source attribution estimates for 28 enteric pathogens. This included the following: recruitment, pre-survey administration, an introductory webinar, elicitation survey administration, a 1-day discussion, readministration of elicitation survey, and a feedback exercise. The pre-survey and elicitation surveys were modeled after previous expert elicitations of enteric illness (Hoffmann et al., 2006; Ravel et al., 2010; Davidson et al., 2011; Vally et al., 2014), and administered via an online survey platform (FluidSurveys: http://www.fluidsurveys.com).

The study occurred between January and April 2014. Ethics approval was granted by Health Canada and PHAC's Research Ethics Board on January 13, 2014 (REB 20130033).

\section{Recruitment}

Snowball recruitment was used to form the expert panel (Garabed et al., 2009). A seed panel of Canadian experts in the areas of food safety, water safety, epidemiology, and surveillance was asked to nominate peers with relevant $\mathrm{Ca}$ nadian expertise. These peers were then asked to nominate additional experts for the panel. Nominated experts were assessed by the study team to ensure representative expertise.

\section{Pre-survey}

An online pre-survey was administered from January 20 to February 25, 2014 to collect background information about experts (Supplementary Appendix A1; Supplementary Data are available online at www.liebertpub.com/fpd). Experts were asked to rank from 1 (low) to 5 (high) their experience with each of 30 enteric pathogens. An algorithm was designed to assign 10 pathogens per expert based on maximizing cumulative self-ranked pathogen experience and assigning pathogens uniformly.

\section{Webinar}

An introductory webinar was presented to experts over 2 weeks in February 2014. Background project information and definitions for transmission routes and point of attribution were provided (Supplementary Appendix A2; Supplementary Tables A1 and A2). The survey tool and a worked example for the survey tool were provided.

\section{Survey tool}

For each pathogen, experts were asked to consider, for 100 domestically acquired cases, how many cases are attributed to each transmission route. The survey prompted experts to produce estimates adding up to 100 across the major transmission routes and $5^{\text {th }}$ and $95^{\text {th }}$ percentiles around those estimates, and to rank their confidence from 1 (low) to 5 (high) for each estimate. Experts were asked if and how their estimates would change if they considered contamination at the kitchen door (i.e., as food or pathogens enter the residential or commercial kitchen) versus at the point of consumption (e.g., of contaminated food or water) (Havelaar et al., 2008). Experts were then prompted to estimate the proportion of cases attributed to a number of foodborne, waterborne, and animal contact transmission subcategories (Butler et al., unpublished data). A sample survey page is presented in Supplementary Appendix A3.

The survey was open from February 17 to March 17, 2014.

\section{Discussion}

Results from the first round of the survey were shared with participants, who were invited to attend a subsequent 1-day discussion on March 20, 2014. At the meeting, preliminary results were reviewed by pathogen and unexpected/unusual results and clusters were discussed.

\section{Survey readministration}

Experts were provided with a summary of the March 20 meeting and points of clarification for issues identified during the discussion, and were asked to consider this new information when revising their estimates. The survey was reopened March 27-April 22, 2014.

\section{Data analysis}

Descriptive statistics were performed on the information collected in the pre-survey. Responses were excluded where experts ranked confidence in their estimates at 1/5 (threshold model); sensitivity analysis of the threshold model versus the complete model (all responses) was performed using Kruskal-Wallis (K-W) tests, which are useful for non-normal data and small sample sizes (Meyer and Seaman, 2013). Spearman's rank correlation was used to test for correlation between parameters. Clustering was explored simultaneously across major transmission routes by pathogen using Ward's minimum-variance method (described in Supplementary Appendix A4). Statistical tests were performed using SAS (SAS 9.3; SAS Institute, Inc., Cary, NC).

Triangular probability distributions were built using @Risk software (Version 6.1.2; Palisade Corporation, Newfield, NY) from best estimate (most likely) and $5^{\text {th }}$ and $95^{\text {th }}$ percentile values from individual estimates, and were combined into cumulative distributions, using Monte Carlo simulation with 10,000 iterations. Akaike's Information Criterion was used to test for the best-fit cumulative probability distribution. Median values and 90\% CrI were calculated from these cumulative distributions; medians are presented as they are less influenced by outliers.

\section{Results}

\section{Recruitment}

Thirty-two experts completed the elicitation survey, and 16 participated in the discussion. Responses for one expert were removed because the survey responses were incorrectly 
Table 1. Median and 90\% Credible Intervals from Cumulative Probability Distributions of Attribution at Point of Consumption for Major Transmission Routes for Each of 28 Enteric Pathogens, and Their Clusters (as Applicable), Where Experts Indicated Confidence in Major Transmission Route Estimates as $>1 / 5$

\begin{tabular}{|c|c|c|c|c|c|c|}
\hline Pathogen & $\mathrm{N}$ & Foodborne & Waterborne & Animal contact & Person-to-person & Other \\
\hline irus & 6 & 9) & $6.1)$ & 7) & 1) & 6.6 \\
\hline Astrovirus & 5 & 9.9 & $.8)$ & $0)$ & 7) & 0.0 \\
\hline Bacillus cereus & 6 & $98.8(88.1-100.0)$ & $0.0(0.0-0.0)$ & $0.0(0.0-0.0)$ & $1.1(0.1$ & $0.1(0.0-0.6)$ \\
\hline Brucella spp. & 5 & $34.6(4.9-64.6)$ & $4.0(0.3-14.9)$ & $54.9(27.6-86.6)$ & $6.6(0.6-17.5)$ & $0.0(0.0-0.0)$ \\
\hline Campylobacter spp. & 12 & $62.3(33.0-81.0)$ & $9.3(2.3-28.1)$ & $15.9(3.5-42.8)$ & $7.7(1.1-27.9)$ & $4.8(0.4-26.6)$ \\
\hline Clostridium botulinum $^{\mathrm{a}}$ & 10 & $65.6(24.1-88.2)$ & $2.6(0.2-10.5)$ & $5.7(0.5-15.9)$ & $0.9(0.0-4.8)$ & $25.1(2.2-67.0)$ \\
\hline Cluster $1^{\mathrm{b}}$ & 8 & $83.3(63.2-91.8)$ & $2.7(0.2-10.7)$ & $6.0(0.5-16.5)$ & $0.9(0.0-5.0)$ & $7.0(0.6-27.2)$ \\
\hline Cluster 2 & 2 & $25.1(17.4-32.6)$ & $0.0(0.0-0.0)$ & $0.0(0.0-0.0)$ & $0.0(0.0-0.0)$ & $74.9(64.8-85.2)$ \\
\hline Clostridium perfringens & 9 & $93.4(50.4-100.0)$ & $2.0(0.1-7.7)$ & $0.9(0.1-3.1)$ & $3.5(0.3-9.3)$ & $0.2(0.0-1.2)$ \\
\hline Cryptosporidium spp. & 11 & $11.3(1.1-37.1)$ & $36.8(13.3-67.6)$ & $23.0(4.9-57.1)$ & $24.2(4.5-61.2)$ & $4.7(0.3-25.7)$ \\
\hline Cyclospora & 13 & $83.1(59.0-93.8)$ & $7.7(0.7-20.5)$ & 3.9 & $4.5(0.4-$ & $0.8(0$. \\
\hline $\begin{array}{l}\text { Escherichia coli, other } \\
\text { diarrheagenic }\end{array}$ & 7 & $41.0(16.1-68.5)$ & 15.6( & 9.9 & 26.4 & $7.1(0$. \\
\hline ETEC & 8 & $\begin{array}{lllll}4 & 11 & 1 & 7\end{array}$ & $15.3(1.7-34.1$ & & & \\
\hline Giardia spp. & 13 & 7.2 & $-75.4)$ & 13.9 & & 7) \\
\hline Hepatitis A & 9 & $29.5(4.8-71.9)$ & $6.2(0.5-26.6)$ & 4.4( & $50.3(12$. & $-31.9)$ \\
\hline Listeria monocytogenes & 13 & $76.5(42.1-89.1)$ & $5.4(0.4-26.2)$ & $6.5(0.5-26.1)$ & $7.3(0.6-26.3)$ & $4.4(0.2-25.7)$ \\
\hline Norovirus & 10 & $18.4(4.0-40.2)$ & $7.4(0.7-22.7)$ & $5.1(0.4-29.6)$ & $65.2(28.9-84.6)$ & $3.9(0.0-24.3)$ \\
\hline Rotavirus & 8 & $7.3(2.1-17.8)$ & $5.9(0.5-18.5)$ & $9.1(0.8-26.2)$ & $77.7(52.8-90.0)$ & $0.0(0.0-0.0)$ \\
\hline $\begin{array}{l}\text { Salmonella spp., } \\
\text { nontyphoidal }\end{array}$ & 15 & $62.9(31.7-79.6)$ & $8.0(0.6-35.0)$ & $12.7(3.0-37.9)$ & $10.0(1.7-36.0)$ & $6.4(0.5-34.6)$ \\
\hline Sap & 4 & & & & & \\
\hline Shigella $\mathrm{s}$ & 11 & 25.9 & $12.2(1.0-39.0)$ & 4.1 & $52.4(22.2-74.0)$ & $5.5(0.4-24.7)$ \\
\hline Staphyloce & 10 & $.1-90.2)$ & $5.3(0.4-26.2)$ & $.5-26.6)$ & $.5-26.2)$ & $4.3(0.0-27.0)$ \\
\hline Toxoplasma gondii & 10 & 51.4 & $-25.5)$ & 33.8 & $7.5)$ & $-11.2)$ \\
\hline Trichinella spp. & 11 & $99.4(53.3-100.0)$ & $0-0.0)$ & $.0-2.9)$ & $0-0.0)$ & $0.0(0.0-0.0)$ \\
\hline VTEC non-O157 & 11 & $59.7(28.4-79.4)$ & $11.4(1.1-32.1)$ & $12.3(2.5-33.4)$ & $10.3(2.2-29.1)$ & $6.2(0.4-37.5)$ \\
\hline VTEC O157 & 11 & $61.4(38.5-79.8)$ & $13.3(3.0-32.1)$ & $9.6(3.6-17.5)$ & $13.2(3.0-32.3)$ & $2.5(0.2-8.3)$ \\
\hline Vibrio parahaemolyticus & 12 & $82.8(46.0-94.6)$ & $11.0(0.9-50.2)$ & $2.0(0.2-6.5)$ & $2.8(0.2-10.6)$ & $1.5(0.1-4.3)$ \\
\hline Vibrio spp., other & 4 & $88.9(82.1-95.5)$ & $7.6(4.7-11.4)$ & $1.8(0.3-3.8)$ & $1.8(0.3-3.8)$ & $0.0(0.0-0.0)$ \\
\hline Vibrio vulnificus & 9 & $70.6(29.5-92.3)$ & $23.2(2.1-62.6)$ & $2.0(0.2-6.3)$ & $4.2(0.4-13.7)$ & $0.0(0.0-0.0)$ \\
\hline Cluster 1 & 7 & $92.8(77.8-99.1)$ & $3.8(0.3-12.6)$ & $1.1(0.1-5.7)$ & $2.3(0.1-9.2)$ & $0.0(0.0-0.0)$ \\
\hline Cluster 2 & 2 & $33.7(26.1-42.8)$ & $57.5(49.6-66.2)$ & $2.6(1.0-4.6)$ & $6.2(2.3-11.6)$ & $0.0(0.0-0.0)$ \\
\hline Yersinia enterocolitica & 13 & $82.8(65.4-95.5)$ & $7.0(0.6-17.5)$ & $6.7(0.6-19.3)$ & $3.6(0.3-10.0)$ & $0.0(0.0-0.0)$ \\
\hline
\end{tabular}

${ }^{\mathrm{a}}$ For Clostridium botulinum and Vibrio vulnificus, median and credible intervals are reported for the pathogen as a whole and for each cluster.

b" "Cluster 1," the larger cluster, is the most appropriate set of estimates to use for both Clostridium botulinum and Vibrio vulnificus, based on biological plausibility of enteric infection. Text presented in gray for pathogen as a whole and for Cluster 2 for C. botulinum and $V$. vulnificus are presented only for comparison and should be disregarded in interpreting these estimates.

ETEC, enterotoxigenic Escherichia coli (E. coli); VTEC non-O157, verotoxin-producing E. coli (VTEC) non-O157; VTEC O157, verotoxin-producing E. coli (VTEC) O157.

entered and they were unavailable for follow-up. Details of recruitment and expert panel composition are presented in Supplementary Appendix A5.

\section{Overview of responses}

Two pathogens (Vibrio cholerae and Salmonella Typhi) were initially included but eliminated from the final phase due to inability of experts to estimate relative contributions of the various transmission pathways. The mean number of experts assigned to each pathogen was 10.3 (range: 5 for sapovirus to 15 for Salmonella spp., nontyphoidal). Mean self-reported expertise ranged from 2.0 for adenovirus to 4.8 for Campylobacter spp. $($ mean $=3.4)$. Experts' average confidence in their estimates ranged from 2.2 for Vibrio spp., other to 4.1 for Trichinella spp. $($ mean $=3.2)$. Overall, confidence was higher in pathogens for which more responses were provided; however, there was no significant correlation found between experts' ranked confidence or expertise and the number of respondents $(|\mathrm{R}|<0.8)$. The threshold model excludes estimates with a confidence of $1 / 5(20 / 286 ; 7 \%)$, and was adopted on the belief that lowconfidence estimates are less likely to represent true values or appropriate confidence intervals. K-W tests showed no significant difference in best estimates between the threshold and complete models $(p>0.05)$. Between 4 and 15 major transmission route estimates per pathogen were included in the final analysis. Correlation between CrI widths and estimate confidence ranking was only significant for foodborne transmission of $B$. cereus $(R=-0.86, p=0.03)$.

For 40/287 (14\%) estimates, experts initially provided incorrect CIs; they were asked to adjust their estimates to include their best estimate. 


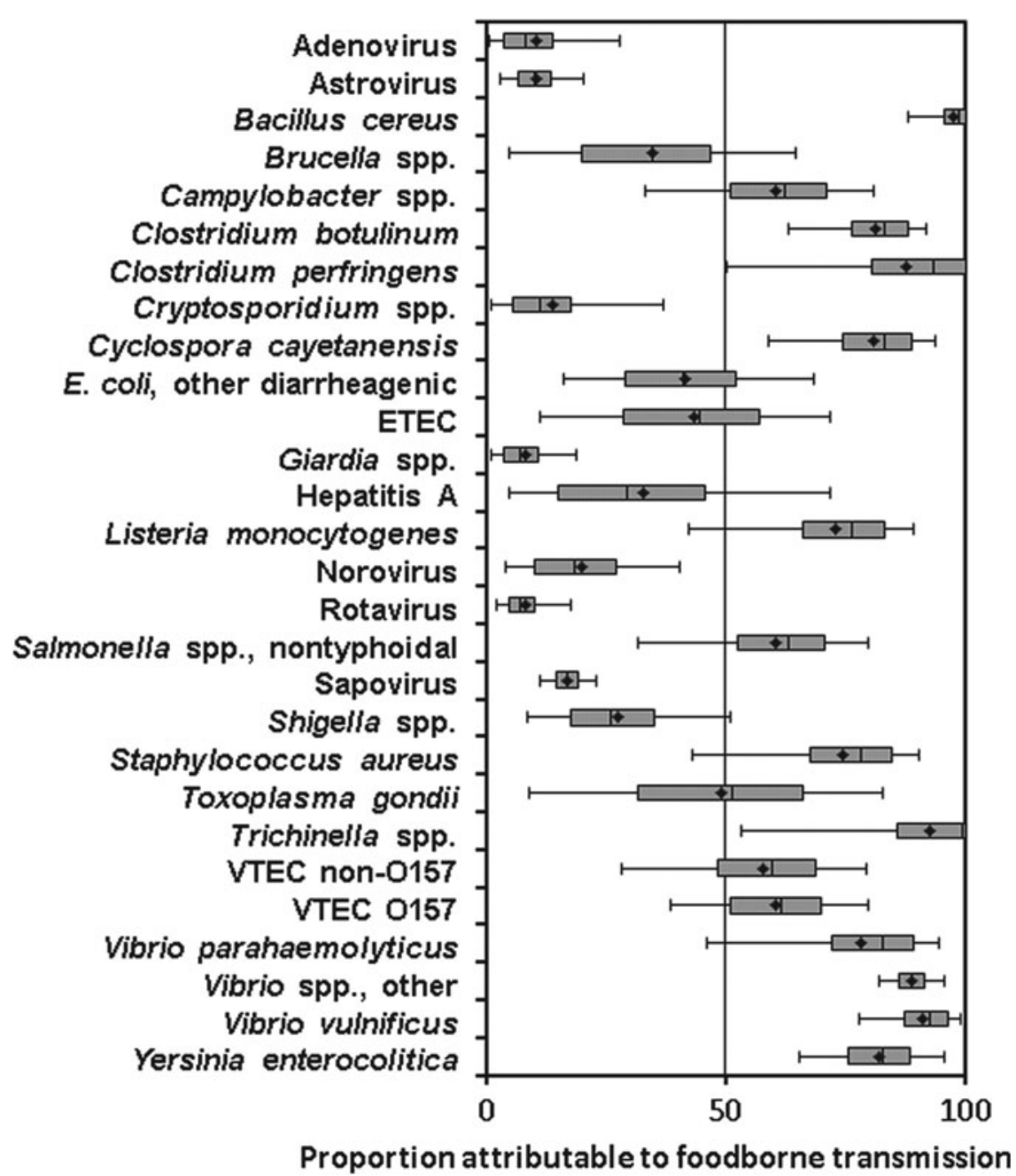

FIG. 1. Box-and-whisker plot of median proportion of transmission via major transmission routes attributed to foodborne transmission for each of 28 pathogens, populated from cumulative probability distributions. The box limits represent $2^{\text {nd }}$ and $3^{\text {rd }}$ quartiles, with whiskers to the $5^{\text {th }}$ and $95^{\text {th }}$ percentile values; diamonds $(\bullet)$ represent mean values. ETEC, enterotoxigenic Escherichia coli; VTEC, verotoxin-producing E. coli.

\section{Revision of responses}

Following the discussion meeting, 26/31 (84\%) experts revised at least 1 of their estimates. After survey readministration, 71/287 (25\%) major transmission route estimates were revised with $0-5$ revisions per pathogen. No significant difference was observed between round 1 and round 2 best estimates (K-W tests, food: $p=0.6$; water: $p=0.3$; animal: $p=0.7$; person-to-person: $p=0.9$; other: $p=0.8$ ). Uncertainty, measured from $90 \% \mathrm{CrI}$ of cumulative distributions, decreased slightly (mean: -0.4 ; range: -2.1 to 1.1).

\section{Clustering}

Significant clustering was observed for Clostridium botulinum and V. vulnificus. On the basis of biological plausibility, the larger clusters of $C$. botulinum $(n=8)$ and $V$. vulnificus $(n=7)$ are considered the most appropriate estimates (Supplementary Figs. A1 and A2). Evidence of clustering for astrovirus and sapovirus was discarded based on nonsignificant differences between distributions across clusters (Supplementary Figs. A3 and A4). Further detail is available in Supplementary Appendix A4.

\section{Estimates of major transmission route attribution}

Cumulative estimates for the attribution of 28 pathogens for the 5 major transmission routes are presented in Table 1. Figures 1-4 present the median best estimates and $90 \% \mathrm{CrI}$ of the 4 transmission routes for each pathogen. The $90 \% \mathrm{CrI}$ widths (Table 1) varied from 0 to 76 cases per 100 domestic cases.

B. cereus, C. perfringens, Cyclospora cayetanensis, Trichinella spp., all 3 Vibrio spp., and Yersinia enterocolitica were mainly attributed to foodborne transmission $(>80 \%)$. For Campylobacter spp., all 4 Escherichia coli categories, Listeria monocytogenes, Salmonella spp., nontyphoidal and Staphylococcus aureus, foodborne transmission remained the main route, but additional routes were also implicated (e.g., Campylobacter spp.: $62.3 \%$ foodborne, $15.9 \%$ animal contact), illustrating the complexity of enteric disease transmission. Waterborne transmission was the dominant route 


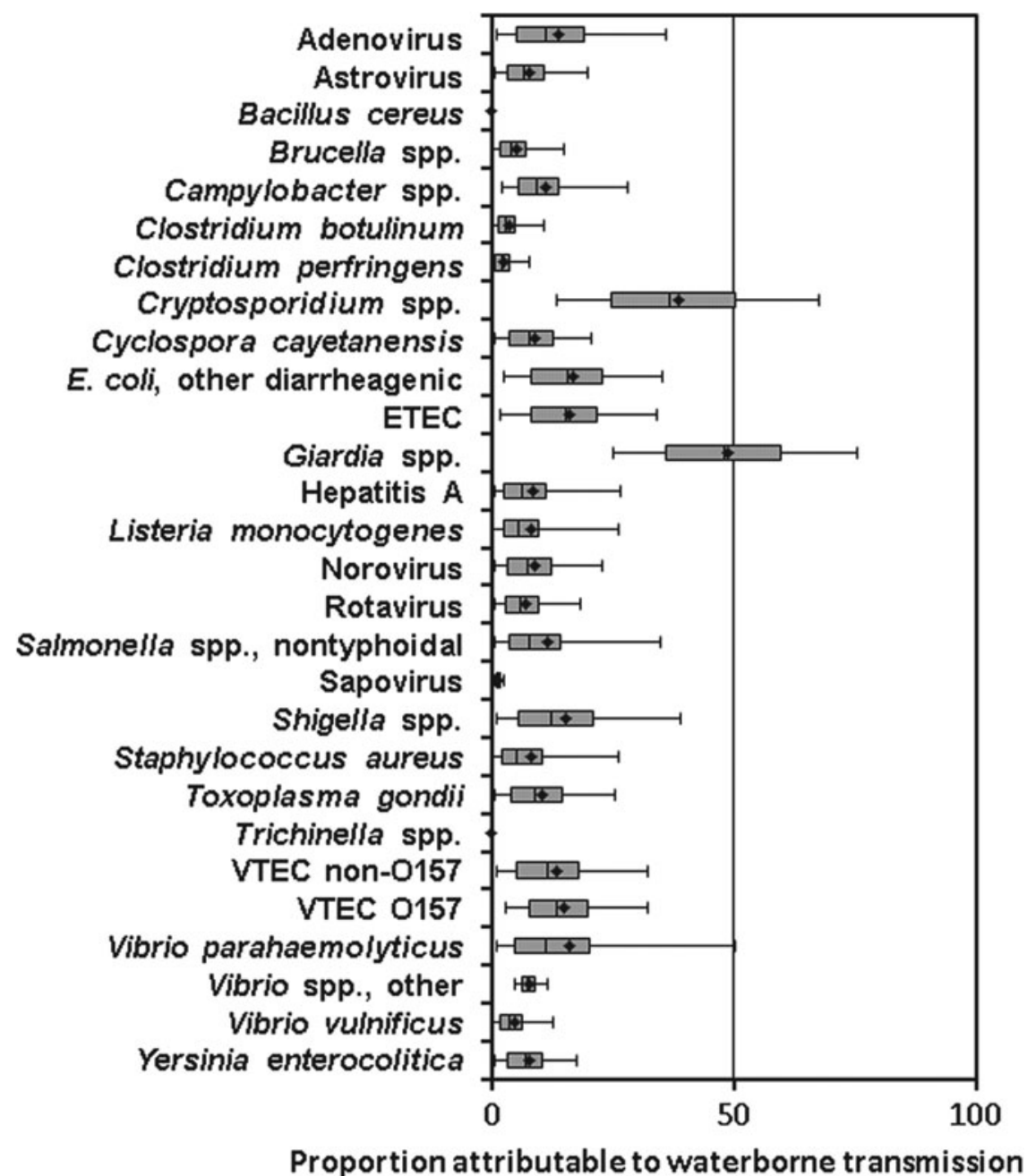

FIG. 2. Box-and-whisker plot of median proportion of transmission via major transmission routes attributed to waterborne transmission for each of 28 pathogens, populated from cumulative probability distributions. The box limits represent $2^{\text {nd }}$ and $3^{\text {rd }}$ quartiles, with whiskers to the $5^{\text {th }}$ and $95^{\text {th }}$ percentile values; diamonds $(\bullet)$ represent mean values. ETEC, enterotoxigenic Escherichia coli; VTEC, verotoxin-producing E. coli.

estimated for Cryptosporidium spp. and Giardia spp. Animal contact was the dominant route estimated for Brucella spp. and Toxoplasma gondii. Person-to-person transmission was the main route for all six viruses and Shigella spp. Up to $10 \%$ of transmission was attributed to "other" routes, suggesting that the four major transmission routes captured (nearly) all enteric transmission.

Experts demonstrated the most uncertainty in producing estimates of Cryptosporidium spp., E. coli (other), enterotoxigenic E. coli (ETEC), hepatitis A, and T. gondii, as measured by CrI width, and higher uncertainty in producing $\mathrm{CrI}$ for foodborne transmission versus other routes.

\section{Kitchen-door estimates}

For 18/28 pathogens, 1-9 experts indicated that their estimates would change if they were to consider attribution at the point of entry into the kitchen (kitchen door) compared to point of consumption. Median and $90 \% \mathrm{CrI}$ were calculated from those who provided kitchen-door estimates and were compared against the point of consumption estimates for the same experts. No kitchen-door estimates were provided for adenovirus, B. cereus, C. botulinum, L. monocytogenes, T. gondii, Trichinella spp., all three Vibrio spp. categories, and Yersinia enterocolitica, and only one estimate was provided for astrovirus, Brucella spp., and C. perfringens.

The majority of the shift in attribution from point of consumption to kitchen door occurs between foodborne and person-to-person transmission. Change in attribution ranged from -7.0 to +56.8 cases for foodborne and -55.7 to +6.8 for person-to-person transmission, measured as the increase in median estimates at point of consumption, compared to kitchen door, where more than 1 estimate was provided. The number of cases attributed to the foodborne route was greater at the point of consumption than the kitchen door for $S$. aureus $(+56.8$, $n=6)$, hepatitis A $(+23.3, n=5)$, ETEC $(+22.3, n=3)$, C. cayetanensis $(+15.7, n=2)$, Shigella spp. $(+15.4, n=9)$, and sapovirus $(+12.8, n=2)$ with concomitant decreases in person-to-person transmission at point of consumption compared with at the kitchen door. Smaller shifts were observed for waterborne $(-4.2$ to +7.2$)$ and animal contact $(-2.7$ to +7.0$)$ transmission between the two points of attribution. 


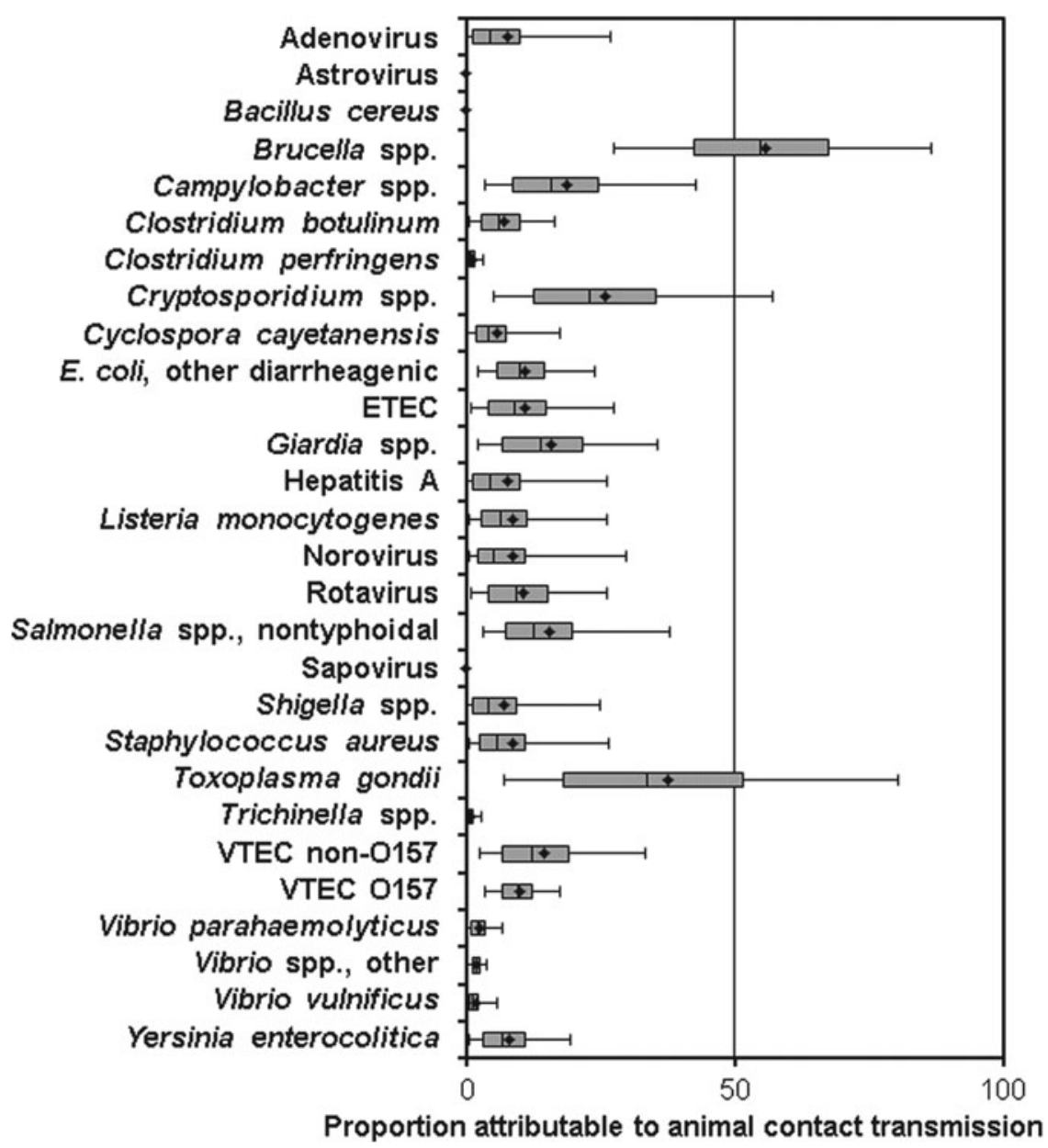

FIG. 3. Box-and-whisker plot of median proportion of transmission via major transmission routes attributed to animal contact transmission for each of 28 pathogens, populated from cumulative probability distributions. The box limits represent $2^{\text {nd }}$ and $3^{\text {rd }}$ quartiles, with whiskers to the $5^{\text {th }}$ and $95^{\text {th }}$ percentile values; diamonds $(\bullet)$ represent mean values. ETEC, enterotoxigenic Escherichia coli; VTEC, verotoxin-producing E. coli.

\section{Discussion}

This expert elicitation aimed to improve the understanding of source attribution of enteric illness for 28 pathogens of public health importance in Canada (FoodNet Canada, 2013). There is limited information in the literature to attribute illness to sources and transmission routes for many of the explored pathogens in Canada.

There is geographic variation in the relative exposure to risk factors within and between countries for a variety of reasons including landscape, climate, environmental factors such as land use, and demographic differences. Examples include cooler average water temperatures in Canada compared to the United States, or dietary risk factors such as raw milk, which is legal in parts of the United States but not in Canada (Health Canada, 2014). Disease rates also vary across countries (e.g., campylobacteriosis incidence in 2012 was 29.3 per 100,000 in Canada [Public Health Agency of Canada, 2014] compared to 14.2 in the United States [CDC, 2014], 101.5 in Australia [Department of Health and Ageing, 2014] and 158.6 in New Zealand [Institute of Environmental Science and Research Ltd., 2013]). This highlights the importance of undertaking source attribution research specific to Canada.
This study was the first to use expert elicitation to attribute such a wide range of pathogens across several major transmission routes. A review of the literature examining similar pathogens and transmission routes using expert elicitation (Table 2) demonstrates convergence of foodborne estimates, despite study location and year of publication, for many pathogens (Cressey and Lake, 2005; Havelaar et al., 2008; Ravel et al., 2010; Scallan et al., 2011; Vally et al., 2014). For example, the current study attributes $99 \%$ of B. cereus to food, compared to $90 \%$ in the Netherlands, $100 \%$ in the United States, and $97 \%$ in New Zealand (Table 2).

The previous Canadian expert elicitation produced estimates of foodborne transmission for 9 of the 28 pathogens explored in this study (Ravel et al., 2010). Mean and median estimates between Ravel et al. (2010) and the current study were similar for Cryptosporidium spp. and Campylobacter spp. (Table 2), and for clusters of L. monocytogenes (previous: 84, current: 76.5) and Y. enterocolitica (previous: 80, current: 82.8). Estimates for Vibrio spp. were also similar, but categorization for Vibrio (V. parahaemolyticus, V. vulnificus, and Vibrio spp., other) differed. Categorizations of E. coli species also varied between the two studies. 


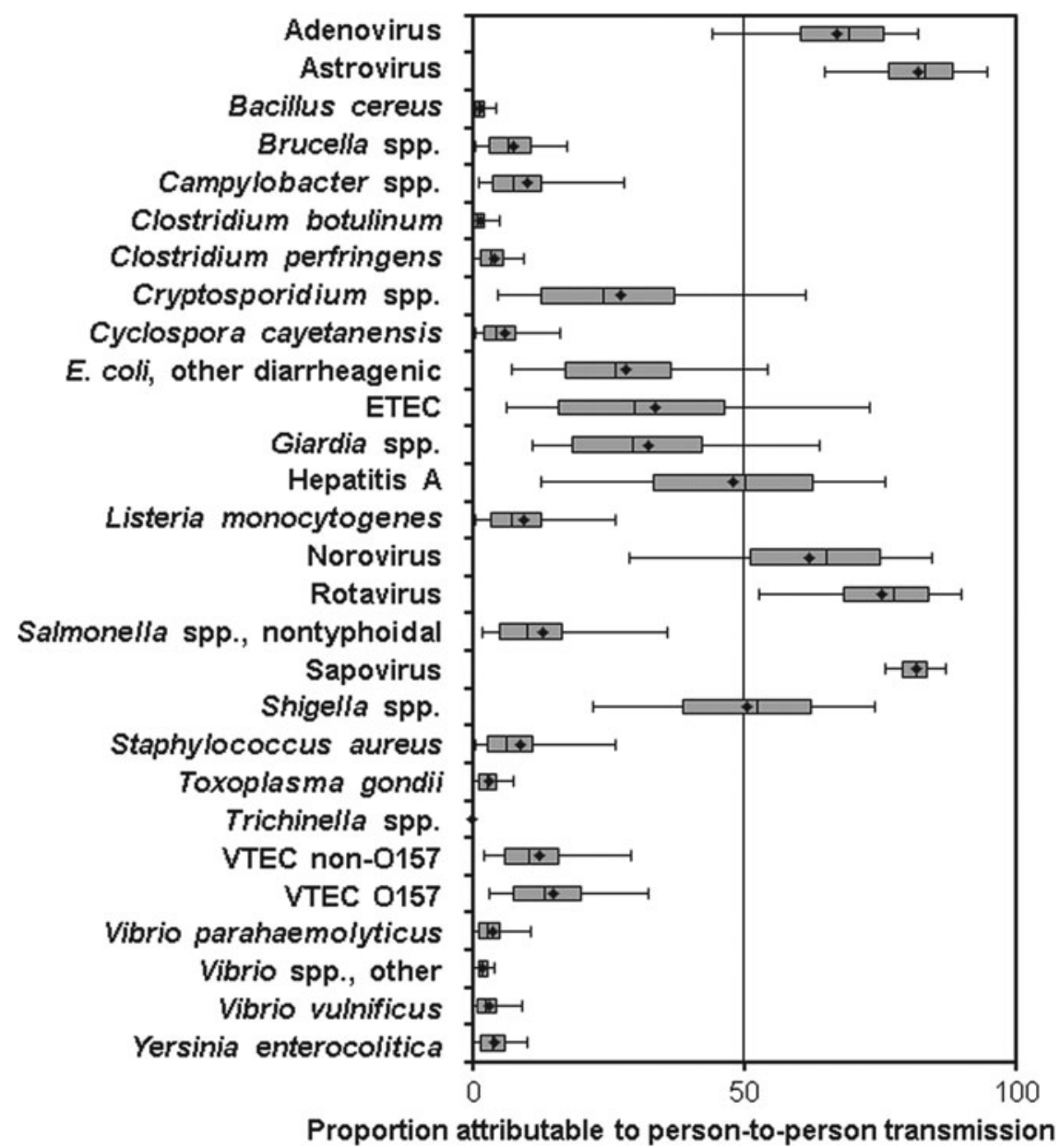

FIG. 4. Box-and-whisker plot of median proportion of transmission via major transmission routes attributed to person-toperson transmission for each of 28 pathogens, populated from cumulative probability distributions. The box limits represent $2^{\text {nd }}$ and $3^{\text {rd }}$ quartiles, with whiskers to the $5^{\text {th }}$ and $95^{\text {th }}$ percentile values; diamonds $(\bullet)$ represent mean values. ETEC, enterotoxigenic Escherichia coli; VTEC, verotoxin-producing E. coli.

Clustering occurred for four pathogens, and in all cases, it is clear which cluster of data should be considered appropriate for the final estimates for these pathogens. For both astrovirus and sapovirus, clustering is assumed to be an artifact of the small number of estimates (five and four responses, respectively; see Supplementary Figs. A3 and A4, Appendix A4). For astrovirus, sapovirus, Brucella spp., Trichinella spp., and the Vibrio spp., experts reported difficulty in providing estimates due to low disease incidence in Canada.

Clustering for $C$. botulinum may have resulted from experts incorrectly considering wound infection (via water) and intravenous drug use. These routes are nonenteric and thus excluded from our analysis. Infant botulism was also mentioned by experts in the smaller cluster. Wound botulism, while an increasingly important transmission pathway in the United States (Sobel, 2005), has never been reported in Canada. Wound infection with $V$. vulnificus is plausible, but is a nonenteric route (Bross et al., 2007).

Inclusion of the meeting between the initial and second rounds of the survey allowed for the opportunity to explore sources of disagreement and potential misinterpretation of study questions. A discussion session was also employed in the Australian elicitation (Vally et al., 2014). During the meeting and in the survey tool, experts were encouraged to provide comments to substantiate their attribution estimates, which were critical for contextualizing findings. The meeting also provided an opportunity to clarify study definitions. For example, the role of congenital infection in transmission of $L$. monocytogenes and $T$. gondii was discussed. In the second survey round, experts were asked to exclude congenital (vertical) transmission from their estimates.

Comparatively few (66 compared to $287 ; 23 \%$ ) estimates were provided for kitchen-door attribution. Experts indicated difficulty estimating these values related to question wording, survey fatigue, or a combination of multiple factors. The shifts in attribution from point of consumption to kitchen door illustrate the importance of contamination by food handlers, especially for $S$. aureus, with the largest increase in foodborne attribution at point of consumption. This shift is biologically plausible; however, the magnitude of these shifts is highly uncertain. The role of cross-contamination and food handling merits further exploration.

The relative contribution of the vehicle versus contamination along the transmission pathway is an important consideration for source attribution. Along the farm-to-fork 
Table 2. Comparison of the Estimated Proportion of Domestic Cases (and Credible Intervals) for 28 Enteric Pathogens Attributed to Foodborne Route to Previously Published Elicitation Studies

\begin{tabular}{|c|c|c|c|c|c|c|}
\hline \multirow[b]{3}{*}{ Pathogen } & $\begin{array}{l}\text { Canada } \\
2014^{\mathrm{a}}\end{array}$ & $\begin{array}{l}\text { Canada } \\
2009^{\mathrm{b}}\end{array}$ & $\begin{array}{l}\text { Australia } \\
2009^{\mathrm{c}}\end{array}$ & $\begin{array}{l}\text { Netherlands } \\
\qquad 2008^{\mathrm{d}}\end{array}$ & U.S. $2007^{\mathrm{e}}$ & $\begin{array}{l}\text { New Zealand } \\
2005^{\mathrm{f}}\end{array}$ \\
\hline & \multicolumn{6}{|c|}{ Data source } \\
\hline & $E E$ & $E E$ & $E E$ & $E E$ & Various $^{\mathrm{g}}$ & $E E$ \\
\hline Adenovirus & $8.3(0.7-27.9)$ & & & & & \\
\hline Astrovirus & $9.9(3.0-20.3)$ & & & & $<1$ & \\
\hline Bacillus cereus & $98.8(88.1-100.0)$ & & & $90(68-100)$ & $100^{\mathrm{h}}$ & $97.4(90.0-98.9)$ \\
\hline Brucella spp. & $34.6(4.9-64.6)$ & & & & 50 & \\
\hline Campylobacter spp. & $62.3(33.0-81.0)$ & $\begin{array}{l}18(6-33)^{\mathrm{i}} \\
68(39-91)\end{array}$ & $76(45-91)$ & $42(16-84)$ & 80 & $57.5(37.1-69.6)$ \\
\hline Clostridium botulinum & $83.3(63.2-91.8)$ & & & & 100 & \\
\hline C. perfringens & $93.4(50.4-100.0)$ & & $97(61-99)$ & $91(72-100)$ & 100 & \\
\hline Cryptosporidium spp. & $11.3(1.1-37.1)$ & $9(0-27)$ & & $12(0-20)$ & 8 & \\
\hline Cyclospora cayetanensis & $83.1(59.0-93.8)$ & & & & 99 & \\
\hline $\begin{array}{l}\text { Escherichia coli, other } \\
\text { diarrheagenic }\end{array}$ & $41.0(16.1-68.5)$ & & $24(4-68)$ & & 30 & \\
\hline ETEC & $44.4(11.1-71.9)$ & & & 100 & & \\
\hline Giardia spp. & $7.2(1.2-18.9)$ & & & $13(0-24)$ & 7 & \\
\hline Hepatitis A & $29.5(4.8-71.9)$ & & $10(2-47)$ & $11(0-20)$ & 7 & \\
\hline Listeria monocytogenes & $76.5(42.1-89.1)$ & $\begin{array}{c}6(0-19) \\
84(59-99)\end{array}$ & $97(63-99)$ & 69 (47-98) & 99 & $27.9(22.2-34.9)$ \\
\hline Norovirus & $18.4(4.0-40.2)$ & $31(5-68)$ & $15(2-59)$ & $17(16-47)$ & 26 & $39.6(27.9-48.9)$ \\
\hline Rotavirus & $7.3(2.1-17.8)$ & & & $13(13-28)$ & $<1$ & \\
\hline $\begin{array}{l}\text { Salmonella spp., nonty- } \\
\text { phoidal }\end{array}$ & $62.9(31.7-79.6)$ & $\begin{array}{l}24(8-44) \\
80(60-95)\end{array}$ & $70(38-88)$ & $55(32-88)$ & 94 & $60.7(45.4-68.9)$ \\
\hline Sapovirus & $16.9(11.3-23.0)$ & & & & $<1$ & \\
\hline Shigella spp. & $25.9(8.6-50.9)$ & $18(0-58)$ & $10(0-50)$ & & 31 & \\
\hline Staphylococcus aureus & $78.4(43.1-90.2)$ & & & $87(73-100)$ & 100 & \\
\hline Toxoplasma gondii & $51.4(8.8-82.7)$ & & & $56(26-88)$ & 50 & $31.5(20.1-41.7)$ \\
\hline Trichinella spp. & $99.4(53.3-100.0)$ & & & & 100 & \\
\hline VTEC non-O157 & $59.7(28.4-79.4)$ & & $54(14-84)$ & $42(21-78)$ & 82 & $39.6(27.0-51.4)$ \\
\hline VTEC O157 & $61.4(38.5-79.8)$ & $\begin{array}{l}14(1-37) \\
76(56-91)\end{array}$ & & $40(15-83)$ & 68 & \\
\hline Vibrio parahaemolyticus & $82.8(46.0-94.6)$ & $\begin{array}{c}2(0-6) \\
93(55-98)\end{array}$ & & & 86 & $89.2(80.0-95.4)$ \\
\hline Vibrio spp., other & $88.9(82.1-95.5)$ & & & & 57 & \\
\hline V. vulnificus & $92.8(77.8-99.1)$ & & & & 47 & \\
\hline Yersinia enterocolitica & $82.8(65.4-95.5)$ & $\begin{array}{l}10(0-32) \\
80(65-92)\end{array}$ & & & 90 & $56.2(41.5-71.8)$ \\
\hline
\end{tabular}

${ }^{\mathrm{a}}$ Current study.

${ }^{\mathrm{b}}$ Ravel et al. (2010).

${ }^{c}$ Vally et al. (2014).

${ }^{\mathrm{d}}$ Havelaar et al. (2008).

${ }^{\mathrm{e}}$ Scallan et al. (2011).

${ }^{\mathrm{f}}$ Cressey and Lake (2005).

${ }^{\mathrm{g}}$ Various: reviews, outbreak reports, case-control studies.

h Bacillus cereus, Clostridium botulinum, C. perfringens, ETEC, and Staphylococcus aureus described as "foodborne" in Scallan et al. (2011).

${ }^{\mathrm{i}}$ Bimodal distributions (clustering) were observed for select pathogens in the previous Canadian elicitation.

EE, expert elicitation; ETEC, enterotoxigenic Escherichia coli; VTEC non-O157, verotoxin-producing E. coli (VTEC) non-O157; VTEC O157, verotoxin-producing E. coli O157.

continuum, there are many factors that influence transmission of pathogens: contaminated irrigation water (Lynch et al., 2009), contamination of carcasses with fecal matter in abattoirs (Nørrung and Buncic, 2008; Rekow et al., 2011), poor adherence to temperature control (Huss et al., 2000; Lynch et al., 2009), and adaptation of pathogens to food processing and antimicrobials (Davidson and Harrison, 2002). Crosscontamination in the kitchen and improper food storage and handling (Papadopoulos et al., 2013) all potentially play a role in human illness (Pires et al., 2009, 2014). Focusing on different points along the continuum will help to inform more specific policy questions and intervention options.

\section{Limitations}

This elicitation was designed to include experts from the public sector, the private sector, and academia; however, the experts were largely from Canadian government organizations (local, provincial, and federal). Fewer experts from academia and industry were nominated by peers. It was 
difficult to find experts who possess an understanding of the broader nature of enteric illness transmission. Despite the inherent difficulty of this approach, 4 to 15 responses per pathogen were elicited.

The results from this study can be used to highlight vulnerabilities to human illness, inform future burden-of-illness studies, and inform food safety policy and research prioritization. Uncertainty in the estimates provided by the expert panel identified key knowledge gaps.

\section{Conclusions}

This article presents the results of an expert elicitation of enteric illness to explore the major transmission routes for 28 pathogens. Expert elicitation is a powerful tool for highlighting uncertainty and producing attribution estimates by pathogen, transmission vehicle, or route. Previous source attribution studies in Canada, the United States, the Netherlands, Australia, and New Zealand have used expert elicitation to understand foodborne transmission. This study explored a broader range of transmission routes (food, water, animal contact, person-to-person, and other) to reflect the spectrum of potential exposures, for a wider range of pathogens than any previous study in Canada or internationally. The results from this study reflect previous findings for some pathogens, while highlighting the continued uncertainty in how viruses are transmitted.

\section{Acknowledgments}

The authors would like to acknowledge Phillip Schmidt (University of Waterloo) and Eddie Ma (University of Guelph) for their contributions to implementing the pathogen selection algorithm. The researchers would like to thank all experts for their participation and engagement with this elicitation study. This research has been conducted under an Natural Sciences and Engineering Research Council of Canada (NSERC) postdoctoral research fellowship held by Ainslie Butler, working with the Centre for Foodborne, Environmental and Zoonotic Infectious Diseases and FoodNet Canada (formerly C-EnterNet).

\section{Disclosure Statement}

No competing financial interests exist.

\section{References}

Batz MB, Hoffmann S, Morris JG Jr. Ranking the disease burden of 14 pathogens in food sources in the United States using attribution data from outbreak investigations and expert elicitation. J Food Prot 2012;75:1278-1291.

Bross MH, Soch K, Morales R, Mitchell RB. Vibrio vulnificus infection: Diagnosis and treatment. Am Fam Physician 2007;76:539-544.

Butler AJ, Thomas MK, Pintar K. A systematic review of expert elicitation methods as a tool for source attribution of enteric illness. Foodborne Pathog Dis 2015;14 (in press).

[CDC] Centers for Disease Control and Prevention. Foodborne Diseases Active Surveillance Network (FoodNet): FoodNet Surveillance Report for 2012 (Final Report). Atlanta, GA: U.S. Department of Health and Human Services, CDC. 2014.

Cressey P, Lake R. Ranking Food Safety Risks: Development of NZFSA Policy 2004-2005. Client Report FW0563:23.
Christchurch, New Zealand: Institute of Environmental Science \& Research Limited, 2005.

Davidson PM, Harrison MA. Scientific status summary: Resistance and adaptation to food antimicrobials, sanitizers, and other process controls. Food Tech 2002;56:69-78.

Davidson VJ, Ravel A, Nguyen TN, Fazil A, Ruzante JM. Food-specific attribution of selected gastrointestinal illnesses: Estimates from a Canadian expert elicitation survey. Foodborne Pathog Dis 2011;8:983-995.

Department of Health and Ageing. National Notifiable Diseases Surveillance System. 2014. Available at: http://www9.health .gov.au/cda/source/cda-index.cfm, accessed September 30, 2014.

FoodNet Canada. FoodNet Canada (formerly known as CEnterNet). 2013. Available at: http://www.phac-aspc.gc.ca/ foodnetcanada/, accessed September 30, 2014.

Garabed RB, Perez AM, Johnson WO, Thurmond MC. Use of expert opinion for animal disease decisions: An example of foot-and-mouth disease status designation. Prev Vet Med 2009;92:20-30.

Havelaar AH, Galindo AV, Kurowicka D, Cooke RM. Attribution of foodborne pathogens using structured expert elicitation. Foodborne Pathog Dis 2008;5:649-659.

Health Canada. Food and Drug Regulations (C.R.C., c. 870). 2014. Available at: http://laws-lois.justice.gc.ca/eng/regulations/ C.R.C.\%2C_c._870/, accessed September 30, 2014.

Hoffmann SA, Fischbeck PS, Krupnick AJ, McWilliams M. Attributing foodborne illnesses to their food sources: Using large expert panels to capture variability in expert judgment. Discussion Paper 06-17-REV. Washington, DC: Resources for the Future. 2006.

Huss HH, Reilly A, Karim Ben Embarek P. Prevention and control of hazards in seafood. Food Control 2000;11:149-156.

Institute of Environmental Science and Research Ltd. Notifiable and Other Diseases in New Zealand: Annual Report 2012. Client Report FW13014. Porirua, New Zealand: The Institute of Environmental Science and Research Ltd., 2013.

Lynch MF, Tauxe RV, Hedberg CW. The growing burden of foodborne outbreaks due to contaminated fresh produce: Risks and opportunities. Epidemiol Infect 2009;137:307-315. Meyer JP, Seaman MA. A comparison of the exact KruskalWallis distribution to asymptotic approximations for all sample sizes up to 105. J Exp Educ 2013;81:139-156.

Nørrung B, Buncic S. Microbial safety of meat in the European Union. Meat Sci 2008;78:14-24.

Papadopoulos A, Vellekoop E, Young I, Pham M, Britten N. Using risk factor weighting to target and create effective public health policy for campylobacteriosis prevention in Ontario, Canada. Am J Pub Health Res 2013;1:32-37.

Pires SM. Assessing the applicability of currently available methods for attributing foodborne disease to sources, including food and food commodities. Foodborne Pathog Dis 2013;10:206-213.

Pires SM, Vieira AR, Hald T, Cole D. Source attribution of human salmonellosis: An overview of methods and estimates. Foodborne Pathog Dis 2014;11:667-676.

Pires SM, Evers EG, van Pelt W, Ayers T, Scallan E, Angulo FJ, Havelaar A, Hald T, Med-Vet-Net Workpackage 28 Working Group. Attributing the human disease burden of foodborne infections to specific sources. Foodborne Pathog Dis 2009;6:417-424.

Public Health Agency of Canada. Notifiable Diseases On-Line. 2014. Available at: http://dsol-smed.phac-aspc.gc.ca/dsolsmed/ndis/charts.php?c $=$ pl, accessed September 30, 2014. 
Ravel A, Davidson VJ, Ruzante JM, Fazil A. Foodborne proportion of gastrointestinal illness: Estimates from a Canadian expert elicitation survey. Foodborne Pathog Dis 2010;7:1463-1472.

Rekow CL, Brashears MM, Brooks JC, Loneragan GH, Gragg SE, Miller MF. Implementation of targeted interventions to control Escherichia coli $\mathrm{O} 157: \mathrm{H} 7$ in a commercial abattoir. Meat Sci 2011;87:361-365.

Scallan E, Hoekstra R, Angulo F, Tauxe R, Widdowson M, Roy S, Jones J, Griffin P. Foodborne illness acquired in the United States-Major pathogens. Emerg Infect Dis 2011;17:7-15.

Sobel J. Botulism. Clin Infect Dis 2005;41:1167-1173.

Thomas MK, Murray R, Flockhart L, Pintar K, Pollari F, Fazil A, Nesbitt A, Marshall B. Estimates of the burden of foodborne illness in Canada for 30 specified pathogens and unspecified agents, circa 2006. Foodborne Pathog Dis 2013; 10:639-648.
Vally H, Glass K, Ford L, Hall G, Kirk MD, Shadbolt C, Veitch M, Fullerton KE, Mustro J, Becker N. Proportion of illness acquired by foodborne transmission for nine enteric pathogens in Australia: An expert elicitation. Foodborne Pathog Dis 2014;11:727-733.

Address correspondence to:

Katarina D.M. Pintar, PhD Centre for Foodborne, Environmental, and Zoonotic Infectious Diseases Public Health Agency of Canada 130 Colonnade Road Ottawa, ON K1A 0K9, Canada

E-mail: katarina.pintar@phac-aspc.gc.ca 